\title{
Analysis of estrogen-regulated genes in mouse uterus using cDNA microarray and laser capture microdissection
}

\author{
Seok Ho Hong ${ }^{1,2}$, Hee Young $\mathrm{Nah}^{2}$, Ji Yoon Lee ${ }^{2}$, Myung Chan \\ $\mathrm{Gye}^{1}$, Chung Hoon $\mathrm{Kim}^{2}$ and Moon Kyoo Kim ${ }^{1}$ \\ ${ }^{1}$ Department of Life Science, College of Natural Sciences, Hanyang University, Seoul 133-791, Korea \\ ${ }^{2}$ Department of Obstetrics and Gynecology, College of Medicine, Ulsan University, Asan Medical Center, Seoul 138-746, Korea \\ (Requests for offprints should be addressed to M K Kim; Email: kimmk@email.hanyang.ac.kr)
}

\begin{abstract}
The steroid hormone, estrogen, plays an important role in various physiological events which are mediated via its nuclear estrogen receptors, ER $\alpha$ and $\operatorname{ER} \beta$. However, the molecular mechanisms that are regulated by estrogen in the uterus remain largely unknown. To identify genes that are regulated by estrogen, the ovariectomized mouse uterus was exposed to $17 \beta$-estradiol (E2) for $6 \mathrm{~h}$ and $12 \mathrm{~h}$, and the data were analyzed by cDNA microarray. The present study confirms previous findings and identifies several genes with expressions not previously known to be influenced by estrogen. These genes include small prolinerich protein $2 \mathrm{~A}$, receptor-activity-modifying protein 3 , inhibitor of DNA binding-1, eukaryotic translation initiation factor 2 , cystatin B, decorin, secreted frizzled-related
\end{abstract}

protein 2 , integral membrane protein $2 \mathrm{~B}$ and chemokine ligand 12. The expression patterns of several selected genes identified by the microarray analysis were confirmed by RT-PCR. In addition, laser capture microdissection (LCM) was conducted to determine the expression of selected genes in specific uterine cell types. Analysis of early and late responsive genes using LCM and cDNA microarray not only suggests direct and indirect effects of E2 on uterine physiological events, but also demonstrates differential regulation of E2 in specific uterine cell types. These results provide a basic background on global gene alterations or genetic pathways in the uterus during the estrous cycle and the implantation period.

Journal of Endocrinology (2004) 181, 157-167

\section{Introduction}

The steroid hormones, estrogen and progesterone, play a pivotal role in the development of the reproductive tract and the regulation of the implantation process. The cellular actions of these steroid hormones are mediated through their ability to bind nuclear receptors, which are basically ligand-activated transcription factors. Estrogen receptors (ERs) are known to exist in at least two types, ER $\alpha$ and $\operatorname{ER} \beta$, in mammals (Green et al. 1986, Kuiper et al. 1996). The estrogen-ER complex directly binds to estrogen response elements on target genes and modulates gene transcription in a ligand-dependent manner, the so-called genomic actions of estrogen (Kousteni et al. 2001). However, some of the actions of estrogen are mediated by intracellular second messengers or various signal-transduction cascades through nongenomic actions (Angel et al. 2000, Ralf \& Martin 2003). Together with the brain, the uterus is a major target of estrogen action, the heterogeneous cell types responding differentially to estrogen. To date, relatively few genes have been identified in the uterus that are regulated by estrogen, with the exact molecular pathways of estrogen and the expression patterns of specific uterine cell types being largely unknown (McMaster et al. 1992, Das et al. 1994, Wang et al. 1994, Diane et al. 2000, Brannvall et al. 2002).

The aim of this study was to identify early and late responsive genes regulated by estrogen in the mouse uterus and to determine whether estrogen-responsive genes are differentially expressed according to the specific uterine cell types. For elucidation of estrogen-responsive genes in the uterus, we carried out cDNA microarray analysis. The technique of cDNA microarray has been used to profile the genome-wide analysis of gene expression in various uterine tissues such as implantation-interimplantation sites, progesterone-treated, and delayed-implanting uterus (Reese et al. 2001, Cheon et al. 2002). We also employed a combination of laser capture microdissection (LCM) and RT-PCR to quantify estrogen-responsive genes in specific cell types of the ovariectomized mouse uterus. Recently, LCM has been used to isolate specific cell types from tissue sections for the purpose of differential gene expression (Green et al. 2003). Using these techniques, we profiled both early and late responsive genes regulated by estrogen and demonstrated that several responsive genes 
were differentially expressed in specific cell types of the estrogen-induced uterus.

\section{Materials and Methods}

\section{Animals and estrogen treatment}

ICR mice were housed within temperature- and lightcontrolled conditions under the supervision of a licenced veterinarian. Mice were maintained on a $12 \mathrm{~h}$ light: $12 \mathrm{~h}$ darkness photoperiod and food and water were available ad libitum. Female mice (6-7 weeks of age) were ovariectomized (OVX) and rested for 14 days before receiving estrogen treatment. They were injected subcutaneously $(0.1 \mathrm{ml} / \mathrm{mouse})$ with sesame oil (control group) alone or with $17 \beta$-estradiol (Sigma, St Louis, MO, USA; $300 \mathrm{ng} /$ mouse; experimental group) dissolved in sesame oil. Mice were killed and uterine horns were collected $6 \mathrm{~h}$ or $12 \mathrm{~h}$ after injection. Uteri collected from both oil-treated and estrogen-treated mice were used for the microarray and LCM studies. Half the uterus was immediately frozen in liquid nitrogen for RNA isolation as described below and the remaining tissue was embedded in Tissue-Tek (Sakura Finetek, Torrance, CA, USA) for LCM. All animal experiments were performed in accordance with the Ulsan University Guide for the Care and Use of Laboratory Animals.

\section{$R N A$ isolation for $\mathrm{cDNA}$ microarray}

Uterine tissues collected from nine female mice in each group were pooled, snap frozen, and homogenized by mortar in liquid nitrogen. For cDNA microarray analysis, total RNA was extracted using TRIZOL reagent (InVitrogen, Carlsbad, CA, USA) and purified using RNeasy total RNA isolation kit (Qiagen, Valencia, CA, USA) according to the manufacturer's instructions. DNA was digested using an RNase-free DNase set (Qiagen) during RNA purification. Total RNA was quantified by spectrophotometer and its integrity was assessed by running on a denaturing $0.8 \%$ agarose gel. Prior to use in cDNA microarray analysis, each uterine RNA sample obtained via the OVX/estrogen treatment/6-h/12-h protocols was validated by assaying for up-regulated or downregulated genes which are known to be regulated by estrogen in the mouse uterus as markers of estrogen responsiveness.

\section{cDNA microarray and data analysis}

To analyze early and late estrogen-responsive genes in the uterus, total uterine RNA was extracted $6 \mathrm{~h}$ and $12 \mathrm{~h}$ after the injection of estrogen to ovariectomized mice (OVX/ estrogen treatment/6-h and 12-h protocol). Profiling of estrogen-regulated gene expression was analyzed with a
TwinChip Mouse-7·4K (Digital Genomics, Seoul, Korea) consisting of 7616 mouse cDNA clones. Each total RNA sample $(20 \mu \mathrm{g})$ was reverse-transcribed with Cy3- (for oil-treated group) or Cy5 (for estrogen-treated group)conjugated dUTP (Amersham Pharmacia Biotech, Piscataway, NJ, USA), using SuperScript II (Gibco BRL, Rockville, MD, USA) and oligo(dT) ${ }_{18}$ primer (Ambion, Austin, TX, USA) in a reaction volume of $20 \mu \mathrm{l}$ according to the method suggested by the manufacturer. After the labeling reaction for $1 \mathrm{~h}$ at $42{ }^{\circ} \mathrm{C}$, unincorporated fluorescent nucleotides were cleaned up using Microcon YM-30 column (Millipore, Bedford, MA, USA). The Cy3- and Cy5-labeled cDNA probes were mixed together and hybridized to a microarray slide. After overnight at $65{ }^{\circ} \mathrm{C}$, the slide was washed twice with $2 \times$ SSC containing $0 \cdot 1 \%$ SDS for $5 \mathrm{~min}$ at $42{ }^{\circ} \mathrm{C}$, once with $0 \cdot 1 \times$ SSC containing $0 \cdot 1 \%$ SDS for $10 \mathrm{~min}$ at room temperature, and finally with $0 \cdot 1 \times$ SSC for $1 \mathrm{~min}$ at room temperature. The slide was dried by centrifugation at 650 r.p.m. for $5 \mathrm{~min}$. Hybridization images on the slide were scanned by Scanarray lite (Packard Bioscience, Boston, MA, USA) and analyzed by GenePix Pro 3.0 software (Axon Instrument, Union City, CA, USA) to obtain gene expression ratios (oil-treated vs estrogen-treated). Logged gene expression ratios were normalized by LOWESS (locally weighted scatter plot smoother) regression (Yang et al. 2002).

The fluorescent intensity of each spot was calculated by local median background subtraction. We used the robust scatter-plot smoother LOWESS function to perform intensity-dependent normalization for gene expression. Scatter plot analysis was made by Microsoft Excel 2000 (Microsoft, Redmond, WA, USA). Significance analysis of microarray (SAM) was performed for the selection of the genes with significant expression changes (Tusher et al. 2001). The statistical significance of the differential expression was assessed by computing a q-value for each gene. To determine the q-value we used a permutation procedure, and for each permutation two-sample $t$-statistics were computed for each gene. Genes were considered differentially expressed when logarithmic gene expression ratios in three independent hybridizations were more than 1 or less than -1 , i.e. a twofold difference in expression level, and when the q-value was $<0 \cdot 1$.

\section{Uterine sections and laser capture microdissection}

Uterine horns were embedded in Tissue-Tek and frozen in liquid nitrogen. Cryosections (thickness, $6 \mu \mathrm{m}$ ) were cut and mounted onto clean glass slides. After the sections were conterstained with Mayer's hematoxylin, each population of uterine cells (luminal epithelial, stromal and muscle cells) was isolated from these sections using P.A.L.M. Robot-Microbeam version 4.0 (P.A.L.M. Microlaser Technologies AG, Bernried, Germany). LCM was performed with a 15-30 $\mu \mathrm{m}$ laser beam, laser power of 
A

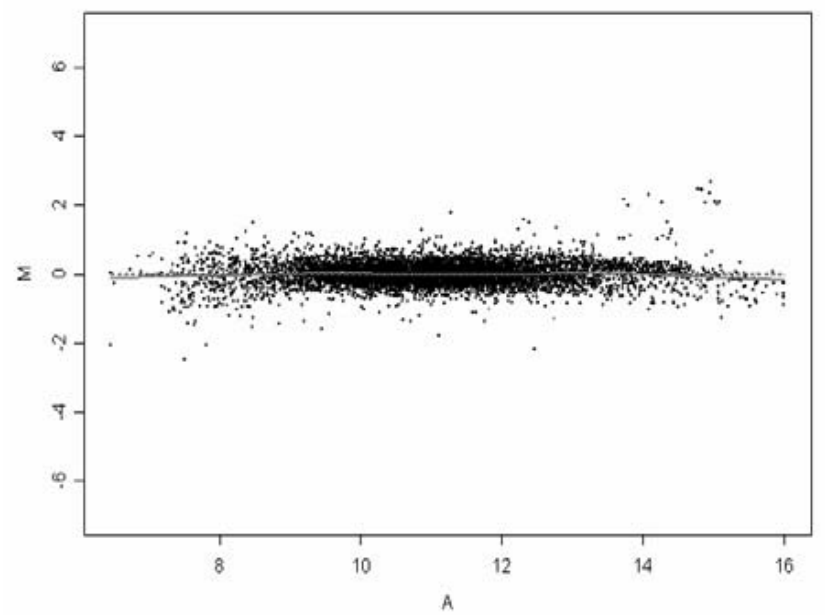

B

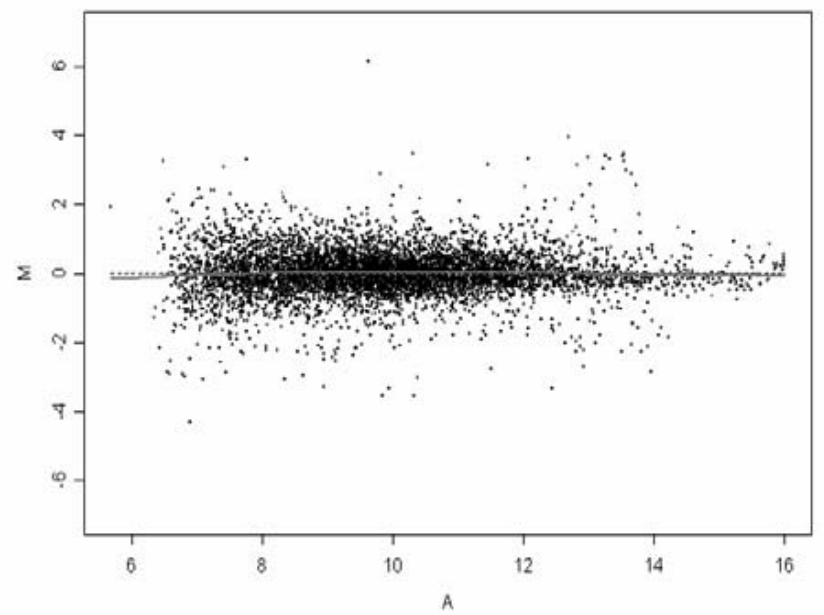

Figure 1 MA-plots ( $M$, expression ratio; $A$, signal intensity) represent genes activated and repressed by estrogen in the ovariectomized mouse uterus. The MA-plot is used to represent the $(R, G)$ data $(R$, red for Cy5; $G$, green for Cy3) where $M=\log 2 R / G$ and $A=\log 2(R \times G)$. (A) OVX/estrogen treatment/6-h protocol, (B) OVX/estrogen treatment/12-h protocol.

$50 \mathrm{mV}$ and a laser power duration of 4-6 ms. For each population of uterine cells, an average of 150 laser shots were transferred onto a $0.5 \mathrm{ml}$ tube cap and stored at $-70{ }^{\circ} \mathrm{C}$ until utilized for total RNA extraction. Single laser shot means an approximate laser spot size of $30 \times 30 \mu \mathrm{m}^{2}$ for luminal epithelial cells and $50 \times 50 \mu \mathrm{m}^{2}$ for stromal and muscle cells.

\section{Confirmation of microarray data with semiquantitative RT-PCR analysis}

Total RNA from whole uterine tissues was extracted using TRIZOL reagent and purified using an RNeasy total RNA isolation kit (Qiagen) following the manufacturer's instructions. One microgram RNA was reversetranscribed at $42{ }^{\circ} \mathrm{C}$ for $60 \mathrm{~min}$ in $20 \mu \mathrm{l}$ reaction mixture consisting of oligo(dT)-adapter primer (Takara, Shiga, Japan) and AMV reverse transcriptase XL (Takara). The following PCR was performed in a total volume of $40 \mu \mathrm{l}$ with $2 \mu \mathrm{l}$ of the $\mathrm{RT}$ reaction mixture, $2 \mu \mathrm{l} 25 \mathrm{mM} \mathrm{MgCl}_{2}$, $4 \mu \mathrm{l} 10 \times$ PCR buffer $(100 \mathrm{mM}$ Tris- $\mathrm{HCl}, 500 \mathrm{mM} \mathrm{KCl}$, $\mathrm{pH} 8 \cdot 3), 4 \mu \mathrm{l} 2.5 \mathrm{mM}$ dNTPs, 10 pmole forward and reverse primer and $1.25 \mathrm{U}$ Taq polymerase (Takara).

Total RNA from LCM-captured luminal epithelial, stromal and muscle cells was extracted using RNeasy mini spin column (Qiagen) as described by the manufacturer. The caps were placed in microcentrifuge tubes with RLT buffer which contains denaturing guanidine isothiocyanate. The captured cell areas were collected by centrifugation at $8000 \times \boldsymbol{g}$ for $3 \mathrm{~min}$. After vortexing and centrifuging the tubes, the caps were removed and RNA was isolated according to the manufacturer's instructions. To remove any potential genomic DNA contamination, an RNase-free DNase set (Qiagen) was used during RNA purification steps. Finally, $24 \mu \mathrm{l}$ column-eluted RNA was all reverse-transcribed at $42^{\circ} \mathrm{C}$ for $60 \mathrm{~min}$ in $40 \mu \mathrm{l} \mathrm{reac}-$ tion mixture consisting of random hexamers (Takara) and AMV reverse transcriptase XL (Takara). The following PCR was performed in a total volume of $40 \mu \mathrm{l}$ with $4 \mu \mathrm{l}$ of the $\mathrm{RT}$ reaction mixture.

The sequences of the primers used in the RT-PCR investigation and the number of cycles (for whole uterus and LCM) tested to assess the best conditions for achieving linear amplification are listed in Table 3. RT-PCR from LCM samples was only performed on a few selected up-regulated genes (Fig. 4). The thermal cycling parameters consisted of denaturing $\left(94{ }^{\circ} \mathrm{C}, 30 \mathrm{~s}\right)$, annealing $\left(60{ }^{\circ} \mathrm{C}, 30 \mathrm{~s}\right)$, and extension $\left(72^{\circ} \mathrm{C}, 30 \mathrm{~s}\right)$. The PCR products were separated by electrophoresis on 1.2\% TBE agarose-ethidium bromide gels and visualized under UV light. The images were quantified by densitometric scanning followed by BioID image analysis software (Vilber-Lourmat, Mama La Vallee, Cedex, France), and gene expression was normalized against the density of the corresponding ribosomal protein L-7 (rpL7) PCR product as internal control.

\section{Results}

Global analysis of estrogen-regulated genes in the ovariectomized mouse uterus

To confirm reproducibility, experiments were repeated independently three times and relative changes were 
Table 1 Genes up-regulated by estrogen in the ovariectomized mouse uterus. Uterine horns were collected $6 \mathrm{~h}(6 \mathrm{H})$ or $12 \mathrm{~h}(12 \mathrm{H})$ after estrogen treatment

\section{Fold change}

\begin{tabular}{|c|c|c|}
\hline $6 \mathrm{H}$ & $12 \mathrm{H}$ & GenBank no \\
\hline$-1 \cdot 15$ & $5 \cdot 48$ & U57635 \\
\hline $1 \cdot 21$ & $3 \cdot 11$ & X14004 \\
\hline $1 \cdot 28$ & $2 \cdot 51$ & ВС002043 \\
\hline $1 \cdot 31$ & $2 \cdot 22$ & AY029181 \\
\hline$-1 \cdot 05$ & $2 \cdot 22$ & ВС029814 \\
\hline $1 \cdot 76$ & $2 \cdot 19$ & ВС014829 \\
\hline $1 \cdot 04$ & $2 \cdot 07$ & AF000294 \\
\hline$-1 \cdot 12$ & $2 \cdot 01$ & M96163 \\
\hline $1 \cdot 59$ & $2 \cdot 89$ & NM011210 \\
\hline $1 \cdot 79$ & $2 \cdot 55$ & BC0027521 \\
\hline $2 \cdot 29$ & $2 \cdot 43$ & AF060091 \\
\hline $1 \cdot 01$ & $2 \cdot 01$ & AF119383 \\
\hline $2 \cdot 11$ & $6 \cdot 12$ & AF209907 \\
\hline $1 \cdot 26$ & $5 \cdot 96$ & ВС020178 \\
\hline $1 \cdot 67$ & $2 \cdot 99$ & ВС015254 \\
\hline$-1 \cdot 19$ & $2 \cdot 94$ & U67610 \\
\hline $1 \cdot 49$ & $2 \cdot 68$ & M63659 \\
\hline$-1 \cdot 17$ & $2 \cdot 57$ & AF228679 \\
\hline $1 \cdot 06$ & $2 \cdot 49$ & BC006613 \\
\hline 1.93 & $2 \cdot 23$ & M55411 \\
\hline $1 \cdot 18$ & $2 \cdot 22$ & ВC046430 \\
\hline $1 \cdot 04$ & $2 \cdot 21$ & U81823 \\
\hline$-1 \cdot 04$ & $2 \cdot 14$ & X77952 \\
\hline $1 \cdot 06$ & $2 \cdot 09$ & ВС021342 \\
\hline $1 \cdot 08$ & $2 \cdot 07$ & AB008792 \\
\hline $1 \cdot 19$ & $2 \cdot 01$ & L78075 \\
\hline $1 \cdot 73$ & $9 \cdot 63$ & X16840 \\
\hline $1 \cdot 44$ & $4 \cdot 03$ & ВС010195 \\
\hline$-1 \cdot 31$ & $3 \cdot 11$ & XM134378 \\
\hline $1 \cdot 19$ & $3 \cdot 02$ & U20344 \\
\hline$-1 \cdot 19$ & $2 \cdot 86$ & AF005772 \\
\hline $1 \cdot 02$ & $2 \cdot 54$ & AF245479 \\
\hline $1 \cdot 29$ & $2 \cdot 31$ & ВС016231 \\
\hline-1.05 & $2 \cdot 29$ & AY099096 \\
\hline$-1 \cdot 04$ & $2 \cdot 28$ & M94087 \\
\hline-1.05 & $2 \cdot 22$ & AK084387 \\
\hline $1 \cdot 16$ & $2 \cdot 21$ & Z22593 \\
\hline $1 \cdot 14$ & $2 \cdot 17$ & Y15001 \\
\hline $1 \cdot 53$ & $2 \cdot 06$ & M64429 \\
\hline $1 \cdot 28$ & $2 \cdot 04$ & BC006871 \\
\hline $1 \cdot 23$ & $2 \cdot 01$ & M31885 \\
\hline $1 \cdot 13$ & $5 \cdot 79$ & L16992 \\
\hline $1 \cdot 21$ & $4 \cdot 74$ & AK013888 \\
\hline $1 \cdot 13$ & $3 \cdot 43$ & AJ309922 \\
\hline $1 \cdot 99$ & $3 \cdot 08$ & XM203179 \\
\hline 1.95 & $75 \cdot 86$ & ВС010818 \\
\hline $1 \cdot 27$ & $12 \cdot 07$ & AY158992 \\
\hline-1.64 & $4 \cdot 11$ & U03419 \\
\hline$-1 \cdot 15$ & $2 \cdot 72$ & X91824 \\
\hline-1.58 & $2 \cdot 39$ & X52046 \\
\hline
\end{tabular}

\section{Gene name}

Cell-cycle-related

Nuclear factor I/C

Protamine 2

Cyclin-dependent kinase inhibior 1A (P21)

Ubiquitin-activating enzyme E1C

FBJ osteosarcoma oncogene

RAN, member RAS oncogene family

Peroxisome proliferator activated receptor binding protein

Serum-inducible kinase

Immune-related

Protein tyrosine phosphatase, receptor type, C

Small chemokine (C-C motif) ligand 11

Proteasome subunit, beta type 5 (Psmb5)

Tumor necrosis factor (ligand) super family, member 13b

Signal transduction-related

Receptor (calcitonin) activity modifying protein 3 (Ramp3)

Serine/arginine-rich protein specific kinase 2

Chemokine orphan receptor 1

Fibroblast growth factor 1

Guanine nucleotide binding protein, alpha 12

Inositol polyphosphate-5-phosphatase, $145 \mathrm{kDa}$

Purinergic receptor P2Y, G-protein coupled 2

Guanine nucleotide binding protein, alpha 11

Debrin-like

Mitogen activated protein kinase 13

Endoglin

MAD homolog 2 (Drosophila)

Phosphatidylinositol 3-kinase, C2 domain containing, gamma

Cell division cycle 42 homolog (S. cerevisiae)

Transcription-related

Homeo box A5

Activating transcription factor 5 (AFT5)

Zinc finger protein 1

Kruppel-like factor 4 (gut)

Paired-like homeodomain transcription factor 3

Williams-Beuren syndrome chromosome region 14

General transcription factor II H, polypeptide 2

CpG binding protein

Activating transcription factor 4 (ATF4)

Paired related homeobox 1

Fibrillarin

loquois related homeobox 3 (Drosophila)

Tripartite motif protein 24

Activating transcription factor 1 (ATF1)

Inhibitor of DNA binding 1 (Id-1)

Enzyme-related

Branched chain keratoacid dehydrogenase E1

Branched chain aminotransferase 1, cytosolic

Mevalonate (diphospho) decarboxylase

Mannosidase 1, beta

Structure-related

Small proline-rich protein 2A (Sprp2A)

Small proline-rich protein $2 \mathrm{H}(\mathrm{Sprp} 2 \mathrm{H})$

Procollagen, type I, alpha 1

Small proline-rich protein A (SprpA)

Procollagen, type III, alpha 1

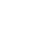

a


Table 1 Continued

\section{Fold change}

\begin{tabular}{rll}
\hline $6 \mathrm{H}$ & $12 \mathrm{H}$ & \multicolumn{1}{c}{ GenBank no. } \\
\cline { 3 - 3 } & & \\
\cline { 3 - 3 } $1 \cdot 87$ & $2 \cdot 79$ & $\mathrm{BC} 034363$ \\
$-1 \cdot 15$ & $2 \cdot 14$ & $\mathrm{AF} 247177$ \\
$1 \cdot 33$ & $2 \cdot 13$ & $\mathrm{C} 26823$ \\
& & $\mathrm{NM} 011182$ \\
$1 \cdot 25$ & $7 \cdot 04$ & $\mathrm{AJ} 011145$ \\
$1 \cdot 52$ & $4 \cdot 77$ & $\mathrm{D} 85904$ \\
$-1 \cdot 19$ & $3 \cdot 38$ & $\mathrm{BC} 002067$ \\
$1 \cdot 77$ & $3 \cdot 11$ & $\mathrm{U} 11027$ \\
$2 \cdot 85$ & $2 \cdot 52$ & $\mathrm{~N} 59807$ \\
$3 \cdot 47$ & $2 \cdot 38$ & \\
$2 \cdot 47$ & $2 \cdot 29$ & \\
\hline
\end{tabular}

calculated. The mean values of the intensities of each spot in the three experiments were calculated, and are plotted in Fig. 1. Of the 7616 genes examined in the OVX/ estrogen treatment/6-h protocol, changes in mRNA expression were detected in 71 genes: 22 were activated and 49 were repressed (Tables 1 and 2). Only $0.9 \%$ of all genes were activated or repressed more than twofold, with estrogen causing no significant differences in the expression of the remaining $99 \cdot 1 \%$ of the genes. However, in the OVX/estrogen treatment/12-h protocol, 638 genes showed a differential expression ratio of more than twofold or less than 50\%: 351 were activated and 287 were repressed. About $8 \cdot 4 \%$ of all genes were activated or repressed (Tables 1 and 2). Out of the 638 activated or repressed genes, 433 genes were listed as known genes in GenBank, and 205 genes were unknown. These genes were classified into eight functional categories based on biological functions: cell-cycle-related, immune-related, signal transduction-related, transcription-related, enzymerelated, structure-related, apoptosis-related, and others (including expressed sequence tags and unknown genes).

\section{Confirmation of estrogen-regulated genes}

The expression patterns of several genes up- or downregulated by estrogen were confirmed to verify the results of the microarray analysis using semiquantitative RTPCR. Several genes were selected in different categories of genes: small proline-rich protein $2 \mathrm{~A}$ (Sprp2A), cystatin B, receptor-activity-modifying protein 3 (Ramp3), proteasome subunit beta type 5 (Psmb5), eukaryotic translation initiation factor 2 (Eif2s2), and inhibitor of DNA binding-1 (Id-1) (up-regulated); and decorin, integral membrane protein 2B (Imp2B), secreted frizzled-related sequence protein 2 (SFR-2), Bach2, chemokine ligand 12 (CXC12), and Id-2 (down-regulated). The primer sets used in the RT-PCR investigation are listed in Table 3 , and the results of semiquantitative RT-PCR analysis are shown in Fig. 2. Densitometric analysis was performed and intensities were normalized to rpL7 for samples on the same gels. Ratios between oil- and estrogentreated groups were calculated for each PCR product, and mean values of relative intensities were then calculated from three different experiments. These data validate the up- or down-regulation of the above selected genes, and are exactly consistent with the microarray expression profiles.

Analysis of selected gene expression patterns in specific uterine cell types using LCM

To further analyze our findings we investigated gene expression of the selected up-regulated genes in specific uterine cell types using the LCM technique. LCM allows the isolation of specific uterine cell types without contamination from other cell types. Green et al. (2003) showed that mRNA from cells could be successfully isolated by LCM from frozen sections with high efficiency, producing RNA with excellent quality and quantity. We isolated populations of specific uterine cell types from frozen sections stained with Mayer's hematoxylin: luminal epithelial, stromal and muscle cells. Figure 3A shows a representative frozen section (stained with hematoxylin) of an ovariectomized mouse uterus, and subsequent microdissections of specific uterine cells - luminal epithelial cells, muscle cells and stromal cells - are shown in Fig. 3B-D respectively. While Sprp2A, Eif2s2, Id-1, and Psmb5 mRNAs were strongly activated only in luminal epithelial cells, Ramp3 and cystatin B were activated in both luminal epithelial and stromal cells (Fig. 4). Our qualitative RT-PCR data demonstrate clearly the differential expression of selected genes in specific uterine cell types. 
Table 2 Genes down-regulated by estrogen in the ovariectomized mouse uterus. Uterine horns were collected $6 \mathrm{~h}(6 \mathrm{H})$ or $12 \mathrm{~h}(12 \mathrm{H})$ after estrogen treatment

\section{Fold change}

$\overline{6 \mathrm{H}} 12 \mathrm{H}$

\section{Gene name}

Cell-cycle-related

Nuclear factor I/A

Epidermal growth factor receptor (EGFR)

Checkpoint kinase 1 homolog (S. pombe)

Topoisomerase (DNA) I

Immune-related

Interleukin 15

Chemokine (C-X-C motif) ligand 12 (CXC-12)

Serine (or cysteine) proteinase inhibitor, Clade G, member 1

Histocompatibility 2, class II antigen E beta

Fc receptor, IgG, alpha chain transporter

Immunoglobulin (CD79A) binding protein 1

Complement component factor $\mathrm{h}$

Proteasome (prosome, macropain) subunit, beta type 9

Chemokine (C-X-C motif) ligand 14 (CXC-14)

Chemokine ( $\mathrm{C}-\mathrm{C}$ motif) receptor 7

Signal transduction-related

Lymphoid blast crisis-like 1

Secreted frizzled-related sequence protein 2 (SFR-2)

Taste receptor, type 1 , member 1

Coagulation factor II (thrombin) receptor

Protein tyrosine phosphatase, receptor type, B

Platelet derived growth factor receptor, alpha polypeptide

F-box and WD-40 domain protein 7, archipelago homolog

Tight junction protein 1

Endothelial differentiation, sphingolipid G-protein

Signal transducer and activator of transcription 1

Interferon-inducible GTPase

Growth factor receptor bound protein 2

Platelet-derived factor, $\mathrm{C}$ polypeptide

Sorting nexin 12

Transcription-related

Neurogenic differentiation 1 (NeuroD)

Transcription factor Dp1

Snail homolog 3 (Drosophila)

Trichorhinophalangeal syndrome I (human)

$\mathrm{Y}$ box protein 2

Histone deacetylase 5

Ring finger protein 12

Eleven-nineteen lysine-rich leukemia gene

Lymphoid enhancer binding factor 1

Inhibitor of DNA binding 2 (Id-2)

Homeo box D9

BTB and CNC homology 2 (Bach2)

WD40 protein Ciao 1

Musculin (Msc)

Enzyme-related

UDP-Gal:betaGlcNAc beta 1,3-galactosyltransferase

Putative phosphatase

Thioether S-methyltransferase

Ubiquitin specific protease 21

Cysteine dioxygenase 1, cytosolic

Structure-related

Decorin

Fibromodulin

Tetranectin (plasminogen binding protein)

Syndecan 2

$\begin{array}{ll}-1 \cdot 04 & -3 \cdot 79 \\ -1 \cdot 33 & -2 \cdot 44 \\ -1 \cdot 44 & -2 \cdot 28 \\ -2 \cdot 11 & -1 \cdot 23 \\ \text { ND } & -7.74\end{array}$

Y07690

BC023729

AF16583

D10061

U14332

BC046827

$-3.19-6.49$

$\begin{array}{ll}-1.54 & -3.63\end{array}$

$-1.23 \quad-3.09$

$1 \cdot 19-2 \cdot 84$

$\begin{array}{ll}-1 \cdot 11 & -2.75\end{array}$

$\begin{array}{ll}-2.19 & -2.65\end{array}$

$-1 \cdot 19 \quad-2 \cdot 16$

$\begin{array}{ll}-1.54 & -2.13\end{array}$

$1 \cdot 21$

$-2 \cdot 13$
$-2 \cdot 04$

$-1 \cdot 46$

$-1 \cdot 41$

$-1 \cdot 29$

$-1.04$

$-1 \cdot 30$

$1 \cdot 23$

$-1.89$

$-1 \cdot 34$

$-1 \cdot 27$

$-1 \cdot 28$

$-1 \cdot 48$

$-1 \cdot 29$

$-1 \cdot 25$

$-1 \cdot 37$

$-9 \cdot 73$

$-6.03$

$-4.63$

$-3 \cdot 37$

$-2 \cdot 91$

$-2 \cdot 81$

$-2 \cdot 63$

$-2 \cdot 57$

$-2 \cdot 41$

$-2 \cdot 29$

$-2 \cdot 25$

$-2 \cdot 24$

$-2 \cdot 04$

$-2 \cdot 03$

$-2 \cdot 28$

$-2.05$

$-1.62$

$-1 \cdot 11$

$1 \cdot 01$

$-1.05$

1.02

$-1 \cdot 14$

$-1.06$

$-1 \cdot 26$

$-1 \cdot 21$

$-1 \cdot 19$

$-1.01$

$-1 \cdot 38$

ND

$-3 \cdot 15$

$-3 \cdot 27$

$-2 \cdot 89$

$-2.63$

$-2 \cdot 41$

$2 \cdot 33$

$-2 \cdot 31$

$-2 \cdot 29$

$-2 \cdot 26$

$-2 \cdot 13$

$-2 \cdot 08$

$-2.07$

$-2 \cdot 04$

BC002026

M36940

D37874

XM196586

NM009888

BC032210

AF192557

L31580

U28495

AF337040

U36757

BC047086

M84607

NM080428

D14340

AB028143

U06924

AF194871

U07617

BC037696

AF062484

BC014722

U28068

X72310

AF133714

AF346836

AF073954

AF207748

AF069992

U80227

X58636

M69293

BC019150

D86604

BC004089

AF087035

$-2 \cdot 49$

$1 \cdot 24$

$-11.97$

$-8.49$

$-3.52$

$-2.99$

$-1.23$

$-1 \cdot 72$

$-2 \cdot 59$

BC046322

U96724

M88694

BC021903

BC013638

$-4 \cdot 75$

$-1 \cdot 37$

$-7 \cdot 03$

X53929

X94998

U08595

U00674 
Table 2 Continued

\section{Fold change}

\begin{tabular}{|c|c|c|}
\hline $6 \mathrm{H}$ & $12 \mathrm{H}$ & GenBank no. \\
\hline$-3 \cdot 25$ & ND & AF161074 \\
\hline$-1 \cdot 55$ & $-2 \cdot 31$ & AF030100 \\
\hline-1.91 & -7.99 & X99807 \\
\hline$-1 \cdot 23$ & $-4 \cdot 18$ & AJ344342 \\
\hline$-1 \cdot 01$ & -3.09 & AY309168 \\
\hline$-2 \cdot 71$ & -2.91 & U76253 \\
\hline$-1 \cdot 02$ & $-2 \cdot 35$ & AB072381 \\
\hline
\end{tabular}

Gene name

Apoptosis-related

Programmed cell death 5 (Pcd5)

Tumor necrosis factor (ligand) superfamily, member 12

Others

Selenoprotein $\mathrm{P}$, plasma, 1

Integrin alpha 9

RNA binding motif protein 5

Integral membrane protein 2B (Imp2B)

Plexin B1

ND, not detected.

\section{Discussion}

Estrogen plays an important role in various physiological events, but the molecular mechanisms that are regulated by estrogen in the uterus remain largely unknown. Therefore, the identification of novel estrogen-regulated gene pathways is essential to understanding how estrogen regulates various aspects of uterine physiological events, such as the estrous cycle and the implantation process. In the present study, we identified genes that are regulated by estrogen in the ovariectomized mouse uterus exposed to estrogen for $6 \mathrm{~h}$ or $12 \mathrm{~h}$ using a cDNA microarray. Prior to use in cDNA microarray analysis, each uterine RNA sample obtained via the OVX/estrogen treatment/6-h/ 12-h protocols was validated by assaying for up-regulated (asparagine synthetase and lactoferrin) or down-regulated (glutathione S-transferase and SFR2) genes which are known to be regulated by estrogen in the mouse uterus

Table 3 Primer sequences for RT-PCR

\begin{tabular}{|c|c|c|c|c|c|}
\hline & & & & Cycles & \\
\hline & & F) and reverse ( $R$ ) primer sequences & bp & Whole & LCM \\
\hline Gene & & & & & \\
\hline Sprp2A & $\mathrm{F}$ & GTGTGGCCTGGGCCTTGTCG & 240 & 22 & 36 \\
\hline & $\mathrm{R}$ & GAGTCGGTGAGCTGGTGAG & & & \\
\hline Cystatin B & $\mathrm{F}$ & CGCGCCATCTGCACAAT & 225 & 22 & 36 \\
\hline & $\mathrm{R}$ & GGCTTGTITCATGGGGGAG & & & \\
\hline Ramp3 & $\mathrm{F}$ & GGTGGTGTGGCGCAGCAAGC & 427 & 24 & 40 \\
\hline & $\mathrm{R}$ & GCAGGGGTCAGGGTCAGGAC & & & \\
\hline Psmb5 & $\mathrm{F}$ & GGCTGGGGTGCAGCGGAT & 350 & 24 & 40 \\
\hline & $\mathrm{R}$ & GGTAGATGGCTCGGCGGG & & & \\
\hline Eif2s2 & $\mathrm{F}$ & CGTGATGGGGGTAAGGAGG & 489 & 24 & 40 \\
\hline & $\mathrm{R}$ & CTAGAGCACAGGTTGGAG & & & \\
\hline $\mathrm{Id}-1$ & $\mathrm{~F}$ & GATCATGAAGGTCGCCAGTG & 476 & 28 & 40 \\
\hline & $\mathrm{R}$ & TCCATCTGGTCCTCAGTGC & & & \\
\hline Decorin & $\mathrm{F}$ & ССССТАССGATGССАGTGTC & 423 & 24 & \\
\hline & $\mathrm{R}$ & GСТCСGTITCAATCCCAGA & & & \\
\hline Imp2B & $\mathrm{F}$ & GTGGCGGTGGATTGCAAGGA & 432 & 22 & \\
\hline & $\mathrm{R}$ & GGGCGGCATACGATGGAAG & & & \\
\hline SFR-2 & $\mathrm{F}$ & TTGGCTTATACGTGCACT & 295 & 24 & \\
\hline & $\mathrm{R}$ & TATTTGAGGGCATCATGCAA & & & \\
\hline Bach2 & $\mathrm{F}$ & СССАТGТСАСАААСССТАТС & 471 & 28 & \\
\hline & $\mathrm{R}$ & TGCTCACCTGACACCGTTCG & & & \\
\hline CXC12 & $\mathrm{F}$ & CGTGGGAGATGCAAGGGCAG & 247 & 22 & \\
\hline & $\mathrm{R}$ & GAGGAGAATGGGGATGAAGC & & & \\
\hline $\mathrm{Id}-2$ & $\mathrm{~F}$ & GTGACCAAGATGGAAATCCT & 236 & 24 & \\
\hline & $\mathrm{R}$ & TTTATTAGCCACAGATAC & & & \\
\hline rPL7 & $\mathrm{F}$ & TCAATGGAGTAAGCCCAAAG & 246 & 22 & 36 \\
\hline & $\mathrm{R}$ & CAAGAGACCGAGCAATCAAG & & & \\
\hline
\end{tabular}



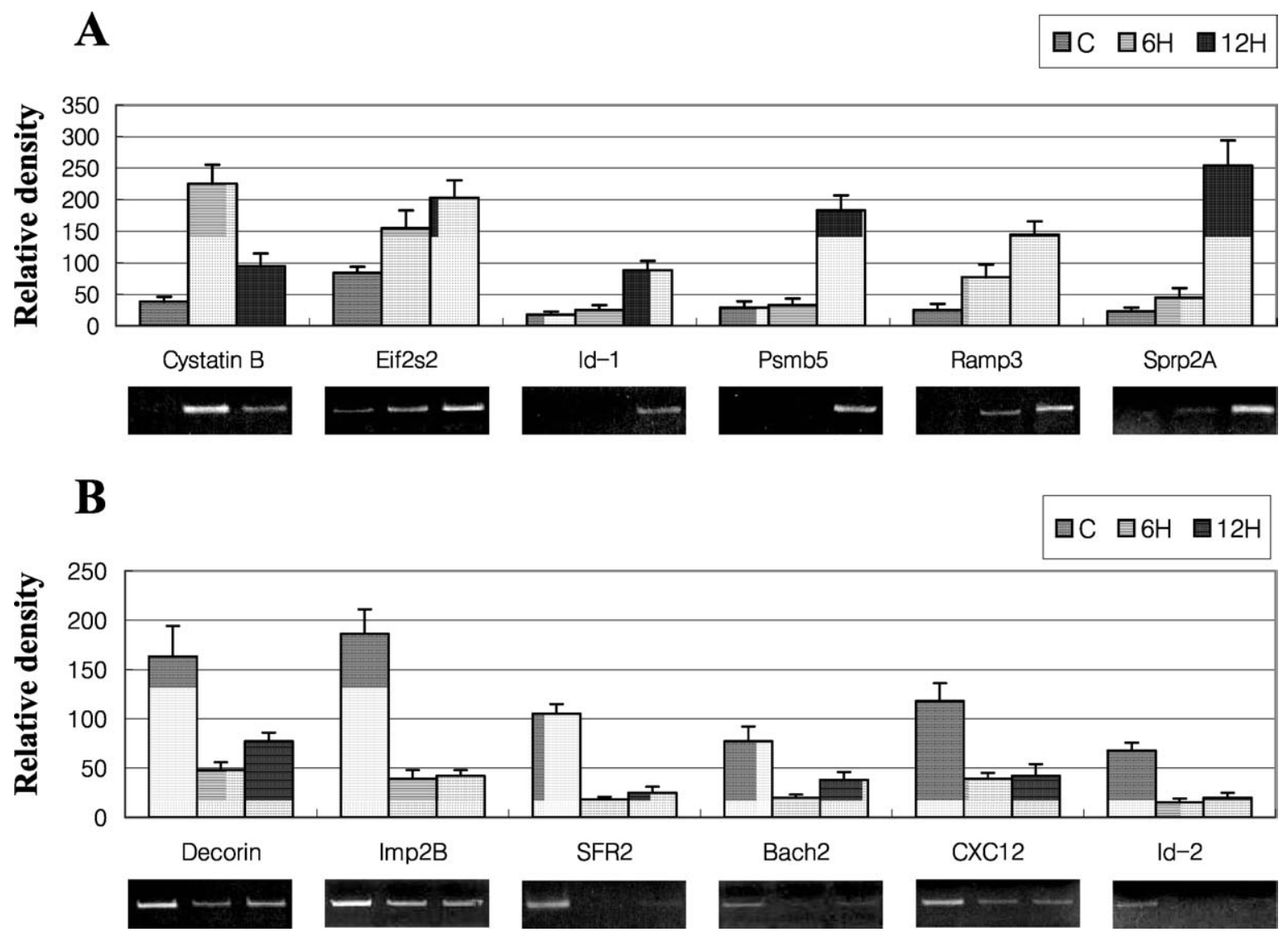

Figure 2 Semiquantitative RT-PCR analysis confirming estrogen-regulated genes. (A) Expression patterns of estrogen-activated genes. Upper panel, representative result of the up-regulated genes. Lower panel, representative result of the down-regulated genes. (B) Expression patterns of estrogen-repressed genes. Values of each band were normalized to rpL7 for the same sample. Data are means \pm S.E.M. from three replicate experiments. C, oil-treated group; $6 \mathrm{H}$, post-estrogen $6 \mathrm{~h} ; 12 \mathrm{H}$, post-estrogen $12 \mathrm{~h}$.

(Das et al. 1998, 2000, Watanabe et al. 2002). Watanabe et al. (2002) listed the early estrogen-responsive genes in the ovariectomized mouse uterus. In their microarray data using the OVX/estrogen treatment/6-h protocol, asparagine synthetase is up-regulated (4-4-fold) and glutathione S-transferase is down-regulated (10-fold). Glutathione S-transferase was the most down-regulated gene by estrogen in their study. Das et al. $(1998,2000)$ also showed the differential regulation of lactoferrin and SFR-2 in response to estrogen in the ovariectomized mouse uterus: while uterine levels of lactoferrin mRNA were strongly enhanced at $12 \mathrm{~h}$ after estrogen administration, SFR-2 levels were reduced over the same period. We therefore used these genes as markers of estrogen responsiveness (data not shown).

Our microarray results showed that only $0.9 \%$ (71 genes) of all genes spotted on the microarray slide were activated or repressed more than twofold in the OVX/ estrogen treatment/6-h protocol, which suggests that direct or fast responses by estrogen are not widespread at the mRNA level in the genes contained in our microarray.
Therefore, if a much larger cohort of cDNAs is used in this experiment, we may obtain a different expression outcome. On the other hand, 638 genes showed a differential expression ratio more than twofold or less than $50 \%$ in the OVX/estrogen treatment/12-h protocol. About $8 \cdot 4 \%$ of all genes were activated or repressed. It is possible that, in addition to direct or genomic pathways, many indirect or nongenomic pathways affected the patterns of mRNA expression in the latter protocol. Watanabe et al. (2002) reported that generally $5 \%$ of all genes were activated or repressed more than threefold by estrogen in an OVX/ estrogen treatment/6-h protocol: 616 genes were determined as estrogen-affected genes, of which 299 were activated and 317 were repressed. It is likely that this difference between their results and ours is due to different strains (they used the C57/BL6/J strain) used in the microarray analysis and the different genes spotted in the microarray techniques.

Even though our results identifying estrogen-regulated genes are quite different from those of Watanabe et al. (2002), two genes did show the same expression pattern: 

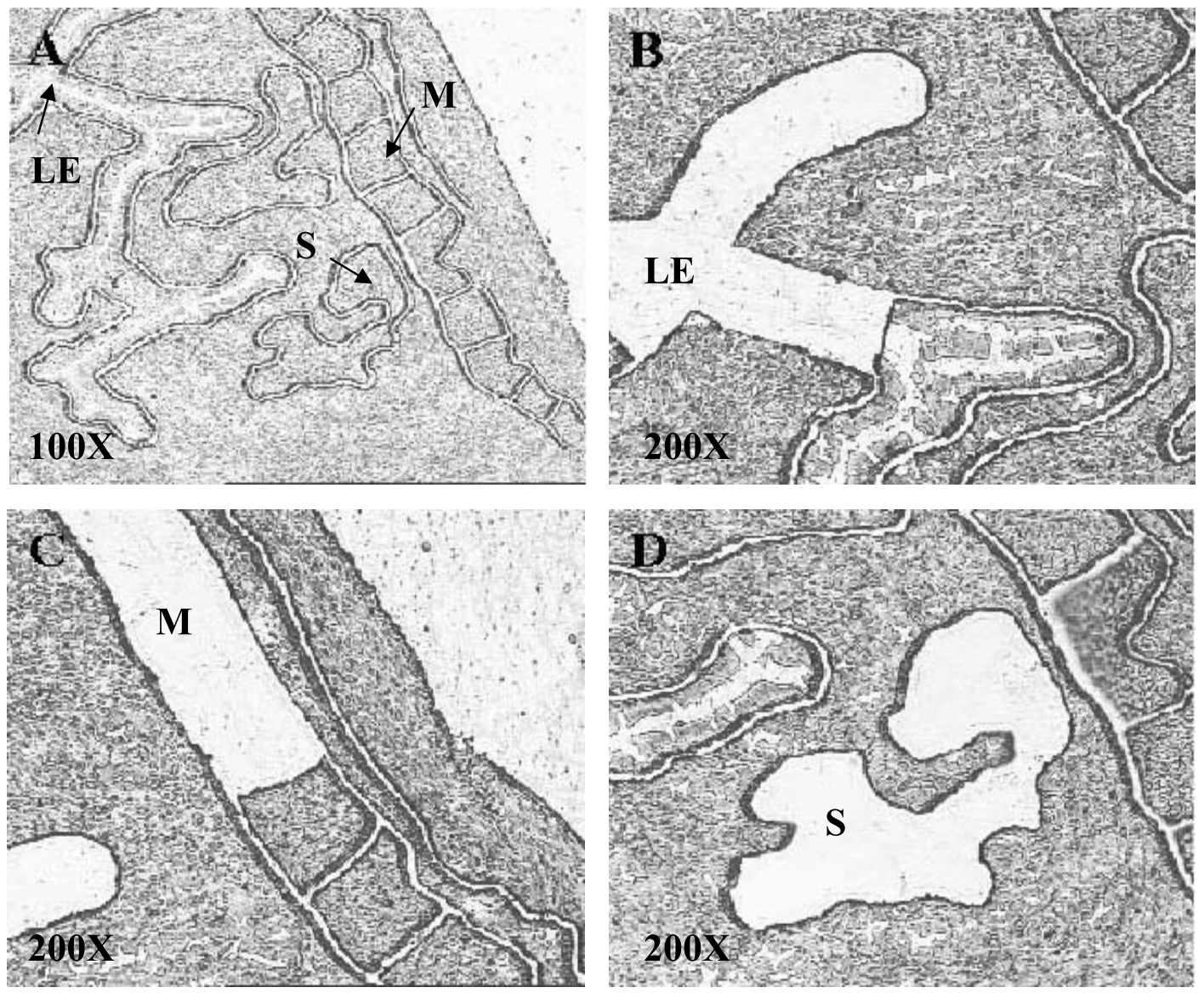

Figure 3 Laser capture microdissection (LCM) procedure. (A) Uterine sections stained with hematoxylin before LCM. LE, luminal epithelial cells; $S$, stromal cells; $M$, muscle cells. Capture sites of each of the uterine cell types are indicated by an arrow. (B-D) Capture of (B) luminal epithelial cells, (C) muscle cells, and (D) stromal cells.

chemokine orphan receptor 1 and Ramp3; they were slightly increased in the OVX/estrogen treatment/6-h protocol and highly increased in the OVX/estrogen treatment/12-h protocol. Calcitonin, calcitonin-generelated peptide, adrenomedullin, and amylin are related hormones and neuropeptides. Ramp-1, Ramp-2 and Ramp-3, through interaction with the calcitonin receptor, ar e required for the recognition of calcitonin-gene-related peptide, adrenomedullin, and amylin by these receptors (Gangula et al. 2000, Fischer et al. 2002). These relationships are enhanced by estrogen, suggesting a role for these genes in uterine estrogen responses.

In the present study, some significant changes in mRNA level were noticed. Activating transcription factors (ATF)-1, ATF-4 and ATF-5 were activated by estrogen in the OVX/estrogen treatment/12-h protocol. It is known that ATF activates gene expression by binding as a homo- or heterodimer to the cAMP response element in the regulatory region of target genes. Although the ATF family is not well characterized in uterine physiological events, such as the estrous cycle and the implantation process, its functions in other tissues suggest an important role in cell proliferation and various signaling mechanisms (Sabbah et al. 1999, Bleckmann et al. 2002). In addition, several genes included in the helix-loop-helix family, such as neuroD, musculin, Id-1, and Id-2, were also identified as estrogen-responsive genes in our microarray analyses. Although the associated changes in mRNA levels were not strongly activated or repressed by estrogen, the estrogenresponsiveness of these genes may indicate their involvement in uterine physiological events based on other recent studies. In the mouse, the main sign of blastocyst attachment sites during the implantation period is angiogenesis. Recent work has shown that the expression of Id-1 and Id-3 is required for correct angiogenesis in the neuroectoderm during embryo development; in particular the partial reduction of Id-1 dosage results in an angiogenic defect in adult mice which blocks the vascularization of tumor xenografts (Lyden et al. 1999). More interestingly, extracellular matrix surrounding endothelial cells was thickened in Id-knockout mice, and thus Id expression may regulate the expression of integrin and matrix metal- 


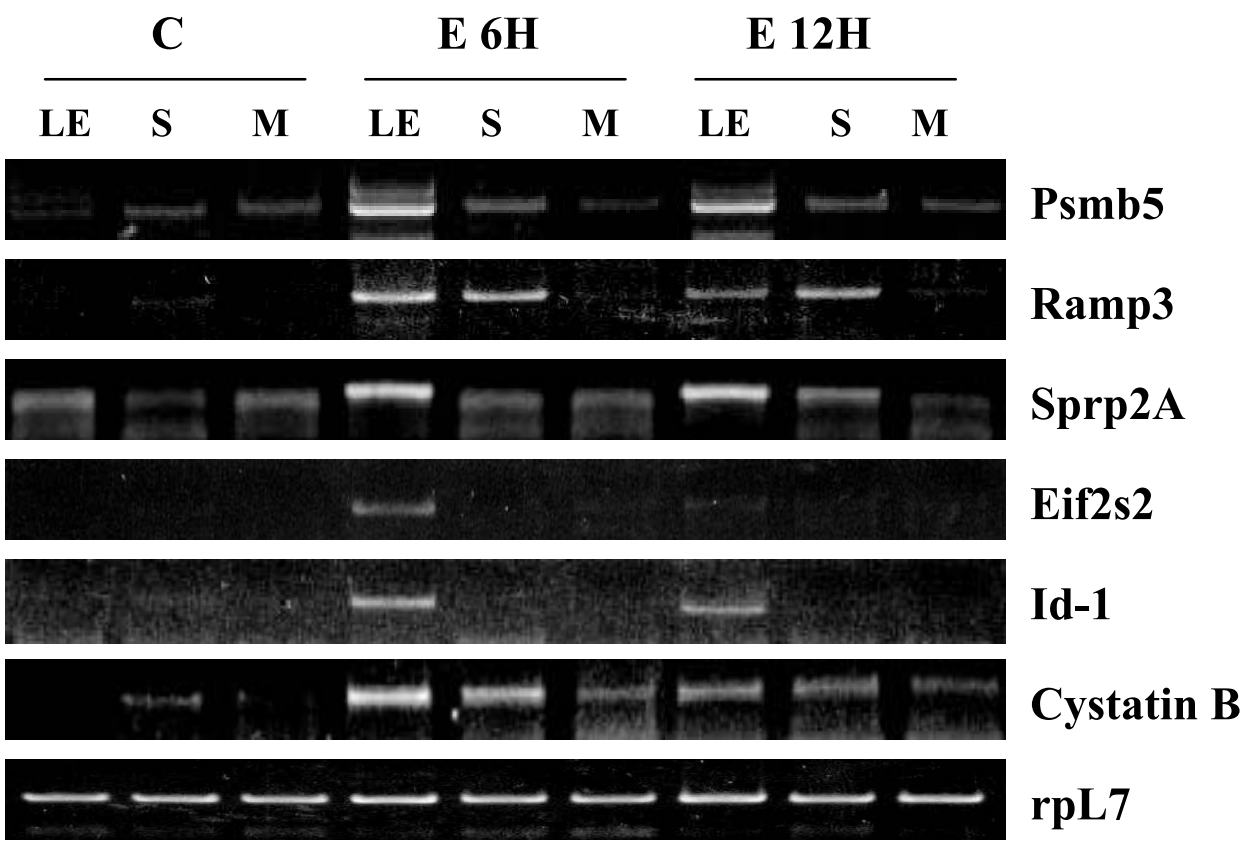

Figure 4 Expression patterns of up-regulated genes by estrogen in specific uterine cell types obtained with LCM. C, oil-treated group; E $6 \mathrm{H}$, post-estrogen $6 \mathrm{~h}$; E $12 \mathrm{H}$, post-estrogen $12 \mathrm{~h}$; LE, luminal epithelial cells; $S$, stromal cells; $M$, muscle cells.

loproteinase MMP2 which are required for tumor angiogenesis. Furthermore, the loss of Id function leads to a decrease in vascular endothelial growth factor expression in endothelial cells (Brooks et al. 1994). These results strongly suggest that endometrial angiogenesis during the estrous cycle or implantation period is directly or indirectly regulated by Id expression under the control of ovarian steroid hormones, prime modulators of cyclic endometrial changes and inducers of local factors produced at the time of implantation.

Interestingly, Sprp2A showed the highest level of upregulation by estradiol in the OVX/estrogen treatment/ $12-\mathrm{h}$ protocol. This gene is reportedly expressed mainly in stratified squamous epithelial cells and controls the differentiation of several cell types (Fischer et al. 1996, Song et al. 1999). To date, three families of Sprps have been described and differentially expressed in various types of epithelia (Kartasova et al. 1996, Fujimoto et al. 1997). Song et al. (1999) isolated 11 mouse Sprp2 genes and examined the expression patterns of Sprp2 (A-K) genes in mouse epithelial tissues. They found that Sprp2A, Sprp2D and Sprp2F were strongly expressed in the uterus, whereas Sprp2C, Sprp2H, Sprp2I, Sprp2J, and Sprp2K were not detected. In our study, Sprp2A mRNA was strongly activated in luminal epithelial cells compared with stromal and muscle cells using LCM techniques. This regulation suggests that the expression of Sprp2A in luminal epithelial cells has a significant effect on the relationship between epithelial cell function and developing embryos. Although clarification of the function of Sprp2A and the significance of estrogen responsiveness in relation to uterine physiological events have not been identified, recent studies have suggested that the Sprp2A gene family plays a pivotal role in the estrous cycle and the implantation process.

To the best of our knowledge this is the first study to profile early and late responsive genes in mouse using cDNA microarray and LCM techniques. We used the LCM technique to isolate specific uterine cell types from frozen sections stained with Mayer's hematoxylin. Using this technique, we determined the differential expression of several up-regulated genes in specific uterine cell types. In conclusion, our results indicate differential spatiotemporal expression of genes regulated by estrogen, and implicate various genes not previously known to be expressed in ovariectomized mouse uterus. Further investigations in regard to progesterone regulation, spatiotemporal expression, protein expression, and the function of these genes may be necessary to understand the exact mechanisms of estrogen action on the estrous cycle and the implantation process.

\section{Acknowledgements}

We thank Prof. Kang Hee-Gyoo in the Department of Biomedical Laboratory Science, Seoul Health College for contributing to several experiments and helpful suggestions. We are also grateful to Lim Hee-Joung 
for the excellent technical assistance of laser capture microdissection.

\section{Funding}

This work was supported by the grant for Asian Institute for Life Sciences (2003-116).

\section{References}

Angel N, Ana BR, Ouahiba L, Marjorie M, Esther F \& Bernat S 2000 Nongenomic actions of estrogens and xenoestrogens by binding at a plasma membrane receptor unrelated to estrogen receptor $\alpha$ and estrogen receptor $\beta$. PNAS 97 11603-11608.

Bleckmann SC, Blendy JA, Rudolph D, Monahan AP \& Schmid SG 2002 Activating transcription factor 1 and CREB are important for cell survival during early mouse development. Molecular Cellular Biology 22 1919-1925.

Brannvall K, Korhonen L \& Lindholm D 2002 Estrogen-receptordependent regulation of neural stem cell proliferation and differentiation. Molecular and Cellular Neurosciences 21 512-520.

Brooks PC, Montgomery AM, Rosenfeld M, Reisfeld RA, Hu T, Klier G \& Cheresh DA 1994 Localization of matrix metalloproteinase MMP2 to the surface of invasive cells by interaction with integrin alpha beta v 3. Cell 79 1157-1164.

Cheon YP, Li Q, Xu X, Demayo FJ, Bagchi IC \& Bagchi MK 2002 A genomic approach to identify novel progesterone receptor regulated pathways in the uterus during implantation. Molecular Endocrinology 16 2853-2871.

Das SK, Tsukamura H, Paria BC, Andrews GK \& Dey SK 1994 Differential regulation of epidermal growth factor receptor (EGF-R) gene and regulation of EGF-R bioactivity by progesterone and estrogen in the adult mouse uterus. Endocrinology 134 971-981.

Das SK, Tan J, Johnson DC \& Dey SK 1998 Differential spatiotemporal regulation of lactoferrin and progesterone receptor genes in the mouse uterus by primary estrogen, catechol estrogen, and xenoestrogen. Endocrionology 139 2905-2915.

Das SK, Tan J, Raja S, Halder J, Paria BC \& Dey SK 2000 Estrogen targets genes involved in protein processing, calcium homeostasis, and Wnt signaling in the mouse uterus independent of estrogen receptor $\alpha$ and $\beta$. Journal of Biological Chemistry 275 28834-28842.

Diane MK, Sylvia CH, Kenneth SK \& Richard PD 2000 Activation of a uterine insulin-like growth factor I signaling pathway by clinical and environmental estrogens: requirement of estrogen receptor-a. Endocrinology 141 3430-3439.

Fischer DF, Gibbs S, Putte P \& Backendorf C 1996 Interdependent transcription control elements regulate the expression of the SPRP2A gene during keratinocyte terminal differentiation. Molecular Cellular Biology 16 5365-5374.

Fischer JA, Muff R \& Born W 2002 Functional relevance of G-protein-coupled-receptor-associated proteins, exemplified by receptor-activity-modifying proteins (RAMPs). Biochemical Society Transactions 30 455-460.

Fujimoto W, Nakanishi G, Arata J \& Jetten AM 1997 Differential expression of human cornifin alpha and beta in squamous differentiating epithelial tissues and several skin lesions. Journal of Investigative Dermatology 108 200-204.
Gangula PR, Wimalawansa SJ \& Yallampalli C 2000 Pregnancy and sex steroid hormones enhance circulating calcitonin gene-related peptide concentrations in rats. Human Reproduction 5 949-953.

Green AR, Edwards RE, Greaves P \& White INH 2003 Comparison of the effect of oestradiol, tamoxifen and raloxifene on nerve growth factor- $\alpha$ expression in specific neonatal mouse uterine cell types using laser capture microdissection. Journal of Molecular Endocrinology $301-11$.

Green S, Walter P \& Kumar V 1986 Human oestrogen receptor cDNA: sequence, expression and homology to v-erb-A. Nature $\mathbf{3 2 0}$ 134-139.

Kartasova T, Darwiche N, Kohno Y, Koizumi H, Osada S, Huh N, Lichti U, Steinert PM \& Kuroki T 1996 Sequence and expression patterns of mouse SPR1: correlation of expression with epithelial function. Journal of Investigative Dermatology 106 294-304.

Kousteni S, Bellido T, Plotkin LI, O’Brien CA, Bodenner DL, Han L, Han K, DiGregorio GB, Katzenellenbogen JA, Katzenellenbogen BS, Roberson PK, Weinstein RS, Jilka RL \& Manolagas SC 2001 Nongenotropic, sex-nonspecific signaling through the estrogen or androgen receptors: dissociation from transcriptional activity. Cell 104 719-730.

Kuiper GG, Enmark E, Pelto-Huikko M, Nilsson S \& Gustafsson JA 1996 Cloning of a novel receptor expressed in rat prostate and ovary. PNAS 93 5925-5930.

Lyden D, Young AZ, Zagzag D, Yan W, Gerald W, O'Reilly R, Bader BL, Hynes RO, Zhuang Y, Manova K \& Benezra R 1999 Id1 and Id3 are required for neurogenesis, angiogenesis and vascularization of tumour xenografts. Nature 401 670-677.

McMaster MT, Teng CT, Dey SK \& Andre GK 1992 Lactoferrin in the mouse uterus: analysis of the preimplantation period and regulation by ovarian steroids. Molecular Endocinology 5 101-111.

Ralf L \& Martin W 2003 Nongenomic actions of steroid hormones. Molecular Cellular Biology 4 46-56.

Reese J, Das SK, Paria BC, Lim H, Song H, Matsumoto H, Knudtson KL, DuBois RN \& Dey SK 2001 Global gene expression analysis to identify molecular markers of uterine receptivity and embryo implantation. Journal of Biological Chemistry 276 44137-44145.

Sabbah M, Courilleau D, Mester J \& Redeuilh G 1999 Estrogen induction of the cyclin D1 promoter: involvement of a cAMP response-like element. PNAS 96 11217-11222.

Song HJ, Poly G, Darwiche N, Lichti U, Kuroki T \& Kartasova T 1999 Mouse Sprr2 genes: a clustered family of genes showing differential expression in epithelial tissues. Genomics 55 28-42.

Tusher VG, Tibshirani R \& Chu G 2001 Significance analysis of microarrays applied to the ionizing radiation response. PNAS $\mathbf{9 8}$ 5116-5121.

Wang X, Das SK, Damm D, Klagsbrun M, Abraham JA \& Dey SK 1994 Differential regulation of heparin-binding epidermal growth factor-like growth factor in the adult ovariectomized mouse uterus by progesterone and estrogen. Endocrinology 135 1264-1271.

Watanabe H, Suzuki A, Mizutani T, Khono S, Lubahn DB \& Iguchi T 2002 Genome-wide analysis of changes in early gene expression induced by estrogen. Genes to Cells 7 497-507.

Yang YH, Dudoit S, Luu P, Lin DM, Peng V, Ngai J \& Speed TP 2002 Normalization for cDNA microarray data: a robust composite method addressing single and multiple slide systematic variation. Nucleic Acids Research 30 E15.

Received 21 November 2003

Accepted 24 December 2003

Made available online as an

Accepted Preprint 12 January 2004 